\title{
Hybrid nanoprobes of bismuth sulfide nanoparticles and CdSe/ZnS quantum dots for mouse computed tomography/fluorescence dual mode imaging
}

\author{
Jun Chen ${ }^{1 \dagger}$, Xiao-Quan Yang ${ }^{1 \dagger}$, Meng-Yao Qin ${ }^{1}$, Xiao-Shuai Zhang ${ }^{1}$, Yang Xuan ${ }^{1}$ and Yuan-Di Zhao ${ }^{1,2^{*}}$
}

\begin{abstract}
Background: X-ray computed tomography (CT) imaging can be used to reveal the three-dimensional structure of deep tissue with high spatial resolution. However, it cannot reveal molecular or cellular changes, and has great limitations in terms of specificity and sensitivity. Fluorescence imaging technology is one of the main methods used for the study of molecular events in vivo and has important applications in life science research. Therefore, the combination of CT and fluorescence imaging is an ideal dual-modal molecular imaging method, which can provide data on both molecular function and tissue structure, and has important research value. In a previous study, $\mathrm{Bi}_{2} \mathrm{~S}_{3}$ nanoparticles were wrapped with quantum dots in $\mathrm{SiO}_{2}$ to generate $\mathrm{CT}$ and fluorescence imaging. However, this type of probe led to low survival and caused innegligible in vivo toxicity in mice. Therefore, it is necessary to develop new multifunctional probes that demonstrate biocompatibility and safety in vivo.
\end{abstract}

Methods: A polyethylene glycol-phospholipid bilayer structure was used to synthesize hybrid clusters containing hydrophobic $\mathrm{Bi}_{2} \mathrm{~S}_{3}$ nanoparticles and quantum dots for combined $\mathrm{CT} /$ fluorescence imaging. Mean particle size, polydispersity index, and zeta potential were used to study the stability over an 8-week test period. In vivo CT and fluorescence imaging experiments were performed, and the in vivo safety of the probe was evaluated, using histopathological, biochemical, and blood analyses.

Results: The probe distinctly enhanced the CT contrast and had fluorescence imaging capability. In addition, the nanocomposite hybrid clusters showed a longer circulation time $(>4 \mathrm{~h})$ than iobitridol. The results also showed that the $\mathrm{Bi}_{2} \mathrm{~S}_{3}-\mathrm{QD} @ D S P E$ probe had good biocompatibility and safety, and did not affect normal organ functioning.

Conclusions: $\mathrm{Bi}_{2} \mathrm{~S}_{3}$-QDDDSPE hybrid clusters exhibited remarkable performance in $\mathrm{CT}$ angiography and fluorescence imaging in vivo.

Keywords: In vivo imaging, Bismuth sulfide, Quantum dots, CT, Fluorescence

\section{Background}

X-ray computed tomography $(\mathrm{CT})$ imaging, a traditional medical imaging technique, yields images with high

\footnotetext{
*Correspondence: zydi@mail.hust.edu.cn

†Jun Chen and Xiao-Quan Yang contributed equally to this work 1 Britton Chance Center for Biomedical Photonics at Wuhan National Laboratory for Optoelectronics - Hubei Bioinformatics \& Molecular Imaging Key Laboratory, Department of Biomedical Engineering, College of Life Science and Technology, Huazhong University of Science and Technology, Wuhan 430074, People's Republic of China

Full list of author information is available at the end of the article
}

spatial resolution. It can be used to determine the threedimensional structure of deep tissue, and to show general morphological characteristics of tissues. It can also provide information on some functional and metabolic features, and has broad applications in life science research and clinical medicine [1-5]. However, it cannot reveal molecular and cellular changes characteristic of biological activity, and has great limitations in terms of specificity and sensitivity. 
Fluorescence imaging technology is one of the main methods used in the study of molecular events in vivo and has important applications in life science research. Therefore, combined CT and fluorescence imaging is an ideal dual-modal molecular imaging technique [6, 7], which provides unified molecular functional and tissue structure imaging, and has important research value [8-10].

In recent years, researchers have used various metallic materials to develop CT imaging probes. Rabin et al. prepared polyvinyl pyrrolidone (PVP)-coated $\mathrm{Bi}_{2} \mathrm{~S}_{3}$ particles which had quasi-rectangular platelet shape in water [5]. The $\mathrm{Bi}_{2} \mathrm{~S}_{3}$-PVP particles had a high $\mathrm{X}$-ray absorption efficiency and a long circulation time in vivo $(>2 \mathrm{~h})$. Chen et al. used a hot injection method to synthesize FePt nanoparticles (FePt NPs), which were effective for CT/MRI dual-mode imaging [11]. Hyeon et al. developed tantalum oxide nanoparticles $\left(\mathrm{TaO}_{\mathrm{x}}\right)$ and Rhodamine $\mathrm{B}$ using an in situ sol-gel reaction and used them for $\mathrm{CT}$ and fluorescence imaging of a rat lymph node [6]. Of these [4-11], bismuth (Bi) appears to be the most promising contrast agent, due to its large atomic number (I, 53; Ta, 73; Pt, 78; $\mathrm{Bi}, 83$ ) and X-ray attenuation coefficient (I, 1.94; Ta, 4.30; Pt, 4.99; Bi, $5.74 \mathrm{~cm}^{2} \mathrm{~g}^{-1}$ at $\left.100 \mathrm{keV}\right)$. However, some problems remain to be solved; for example, it is difficult to control the size of $\mathrm{Bi}_{2} \mathrm{~S}_{3}$ nanoparticles, and their surface is also difficult to modify, thus hindering their application as CT-based multifunctional probes. It is for this reason that use of a Bi contrast agent with an optical probe has seldom been reported. However, a variety of fluorescent materials have been used in fluorescence imaging, for example, fluorescent dyes and quantum dots (QDs). Compared with fluorescent dyes, which have obvious disadvantages in direct biological imaging, QDs show higher brightness and photo-stability, broad excitation profiles, narrow/symmetric emission spectra, and high quantum yields, and are therefore suited for use in many areas of biomedical imaging research.

In a previous study [12], we wrapped $\mathrm{Bi}_{2} \mathrm{~S}_{3}$ nanoparticles with quantum dots in $\mathrm{SiO}_{2}$. These composite nanoparticles showed promise for use in $\mathrm{CT}$ and fluorescence imaging in vitro and in vivo; however, they also showed in vivo toxicity for mice and led to a low survival rate. 1,2-Dipalmitoyl-sn-glycero-3-phosphoethanolamine-N[methoxy(polyethylene glycol)-2000] (PEG-DSPE) has been used by some groups to prepare in vivo probes. For example, Sailor et al. used PEG-phospholipid to prepare hybrid nanoparticles that contained magnetic iron oxide nanoparticles, QDs, and the anticancer drug doxorubicin for simultaneous targeted drug delivery and dual-mode near-infrared fluorescence imaging and MRI of diseased tissue in vitro and in vivo [13]. Lu et al. used PEG 2000DSPE to coat Yb-based nanoparticles as a high-performance CT contrast agent for in vivo angiography and bimodal image-guided lymph node mapping, and they found that the gradual enhancement of signals for the liver and spleen continued for $>2 \mathrm{~h}$ [14]. In the present study, PEG 2000-DSPE was used to synthesize hybrid clusters containing hydrophobic $\mathrm{Bi}_{2} \mathrm{~S}_{3}$ nanoparticles and quantum dots for combined $\mathrm{CT} /$ fluorescence imaging (Fig. 1). For CT imaging of the liver and spleen, the particles produced a clear contrast enhancement after $30 \mathrm{~min}$,

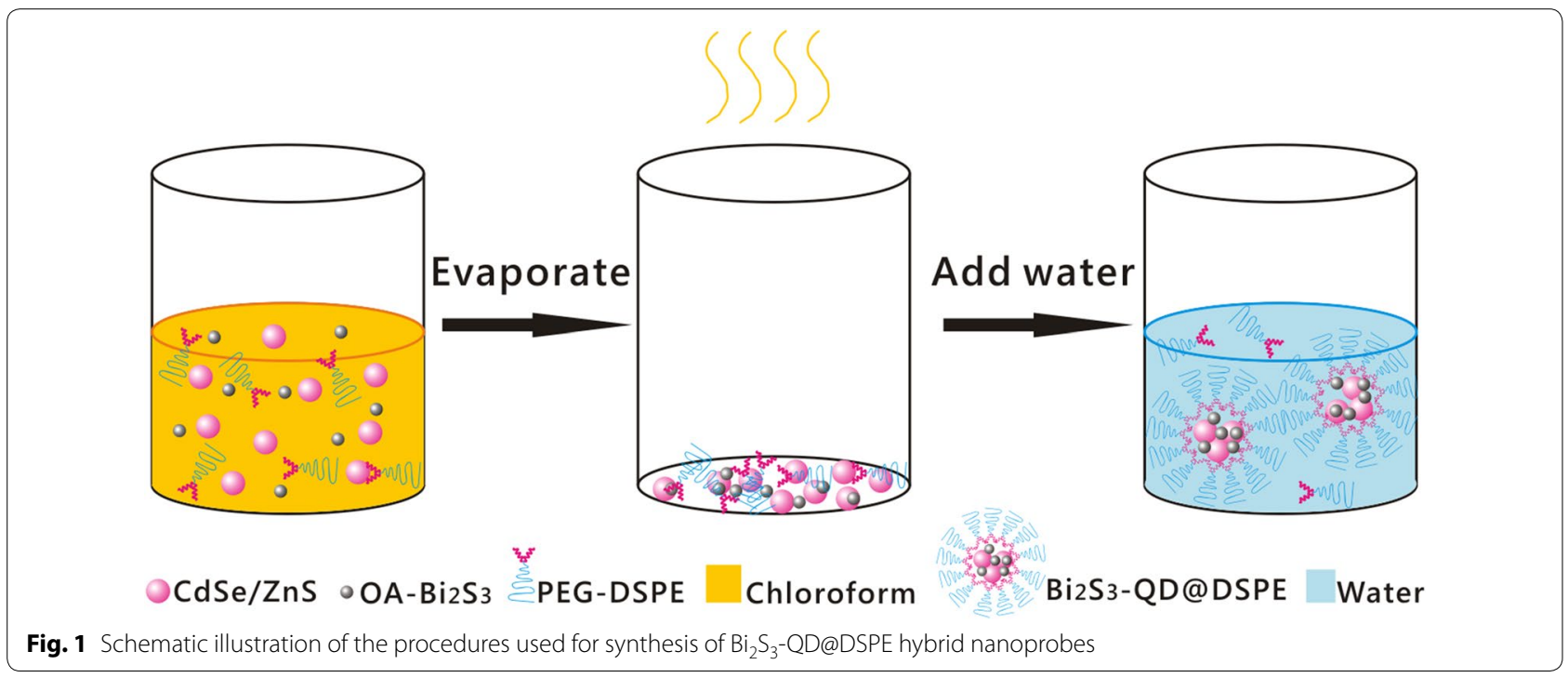


and the enhancement continued for $>4 \mathrm{~h}$; for fluorescence imaging, the liver and kidneys continued to show fluorescence after $24 \mathrm{~h}$, indicating that the probes have a long circulation time. The results also showed that the $\mathrm{Bi}_{2} \mathrm{~S}_{3}$-QD@DSPE probes show good biocompatibility and safety, and did not affect normal organ functioning. Thus, our results show that $\mathrm{Bi}_{2} \mathrm{~S}_{3}$-QD@DSPE hybrid clusters exhibit remarkable performance in $\mathrm{CT}$ angiography and fluorescence imaging in vivo.

\section{Results and discussion}

To synthesize $\mathrm{Bi}_{2} \mathrm{~S}_{3}$-QD@DSPE, we first prepared hydrophobic $\mathrm{Bi}_{2} \mathrm{~S}_{3}$ nanoparticles and QDs. The photoluminescence (PL) spectra of the QDs showed that they had an emission wavelength of approximately $650 \mathrm{~nm}$ (Fig. 2a) with a full-width-at-half-maximum (FWHM) of $28 \mathrm{~nm}$. TEM images showed that these hybrid clusters were $30-40 \mathrm{~nm}$ in diameter (Fig. 2b), which was in good agreement with the average diameters determined by dynamic light scattering measurements (Fig. 3a). After the synthesis of $\mathrm{Bi}_{2} \mathrm{~S}_{3}$-QD@DSPE, the stability of hybrid clusters was evaluated by examining changes in the mean particle size, polydispersity index (PDI), and zeta potential during storage at 4, 25, and $37{ }^{\circ} \mathrm{C}$. Figure 3 illustrates that the mean size, PDI, and zeta potential of the probes were approximately $50 \mathrm{~nm}$, 0.188 , and $-35.7 \mathrm{mV}$, respectively, and the probes did not show any significant changes in particle size, PDI, or zeta potential when maintained at 4,25 , and $37^{\circ} \mathrm{C}$ for the 8-week test period, indicating that the probes show sufficient stability. The probes showed a negative zeta potential due to dissociation of the phosphate group in the PEG-phospholipid moiety, consistent with previous reports $[15,16]$.

Various concentrations of $\mathrm{Bi}_{2} \mathrm{~S}_{3}$-QD@DSPE were dispersed in deionized water. As the concentration of $\mathrm{Bi}_{2} \mathrm{~S}_{3}$ QD@DSPE increased, the Hounsfield unit (HU) values obtained using the in-house-built CT system increased linearly (Fig. 4a, b). The probes showed higher $\mathrm{HU}$ values and CT contrast effects than the same concentration of iopromide.

To study their biodistribution, $\mathrm{Bi}_{2} \mathrm{~S}_{3}$-QD@DSPE particles were injected into the tail veins of mice. Distribution of the probes was tracked by X-ray CT imaging before injection and at $30 \mathrm{~min}, 1,2,4$, and $24 \mathrm{~h}$ after injection. Figure 5a shows that CT contrast of the heart (red arrow) was enhanced after $30 \mathrm{~min}$ and decreased $1 \mathrm{~h}$ after administration of $\mathrm{Bi}_{2} \mathrm{~S}_{3}$-QD@DSPE, likely because the probes spread to the heart and then to other organs with blood circulation. Serial CT sectional and coronal views (Fig. 5b-d) revealed that the liver (orange arrow) and spleen (yellow arrow) showed clear contrast enhancement after $30 \mathrm{~min}$, and that the enhancement continued for $>4 \mathrm{~h}$, indicating that the probes show a long circulation time. However, contrast was weak after $24 \mathrm{~h}$, which may imply that most of the probes were eventually metabolized and did not accumulate in vivo. Furthermore, the HU values of organs at several time points were tested (Table 1). After injection, the HU value of the heart began to peak at approximately $30 \mathrm{~min}$, and spleen and liver signal intensity increased until they peaked at 1-2 h (spleen: 107.9-513.4; liver: 123.1-257.1). The contrast effect in all of the examined organs returned to normal levels after $24 \mathrm{~h}$, as shown in the serial CT images.
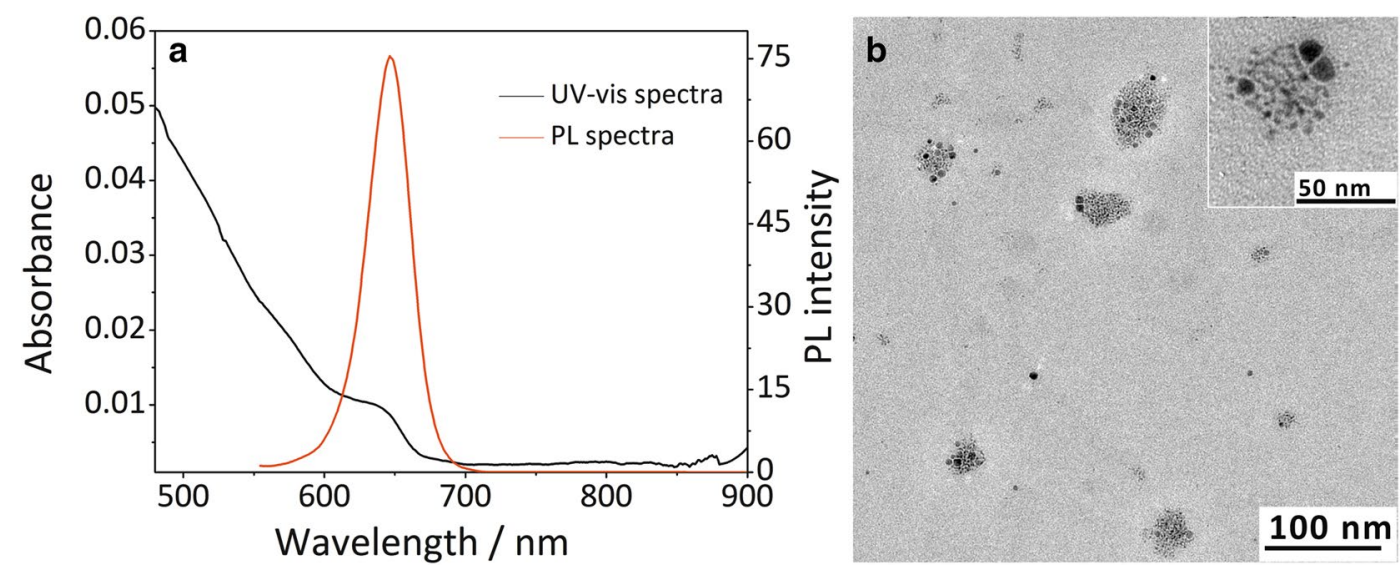

Fig. 2 UV-vis absorption spectra and fluorescence spectra of QD (a) and TEM image of $\mathrm{Bi}_{2} \mathrm{~S}_{3}-\mathrm{QD} @ D S P E$ hybrid clusters (b) 


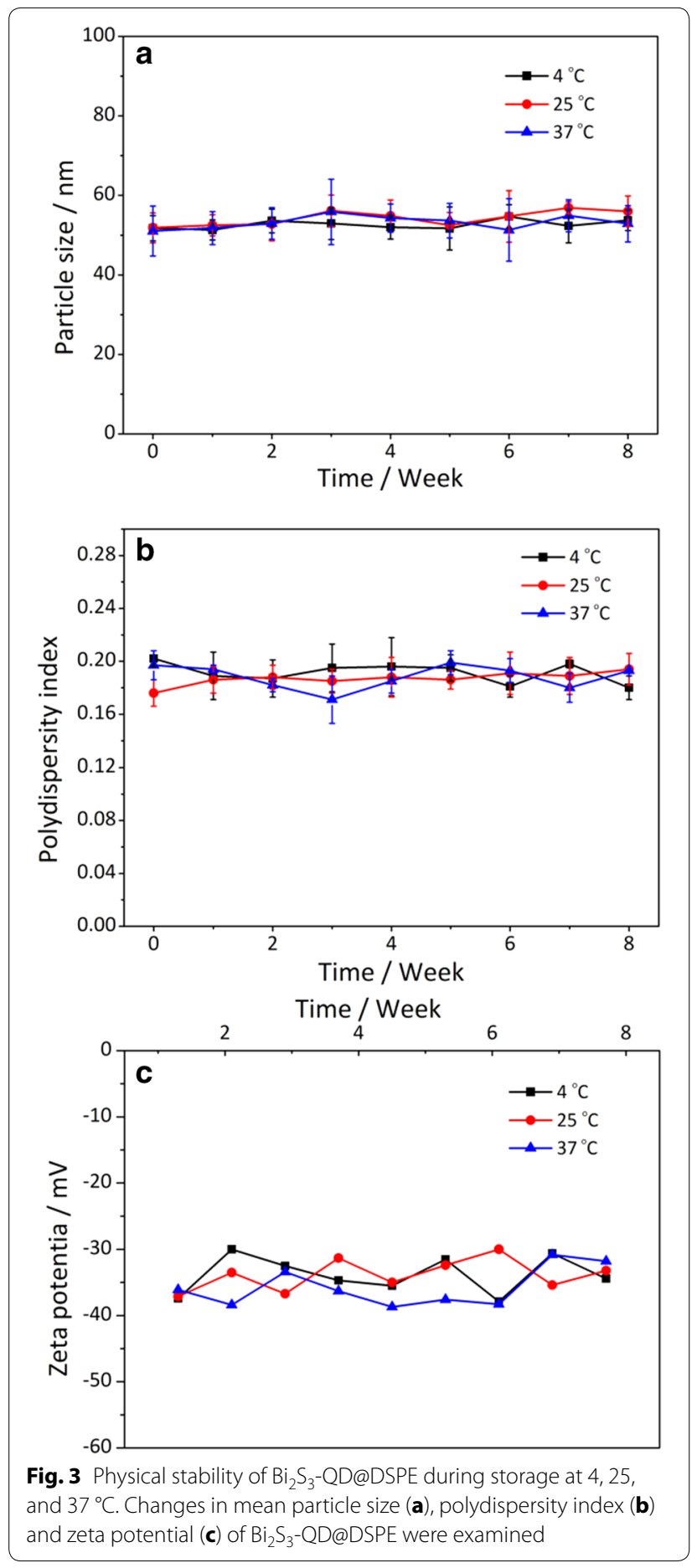

The excitation wavelength of our in house-built imaging system for $\mathrm{Bi}_{2} \mathrm{~S}_{3}$-QD@DSPE was $500 \mathrm{~nm}$ and showed limited optical imaging capability in vivo, and therefore the mice were sacrificed after CT imaging. In this study, the auto-fluorescence signal has been deducted from all of the results for fluorescence imaging. Distribution of the probes was tracked by fluorescence imaging at 1,2, 4, and $24 \mathrm{~h}$ after injection. Figures 6 and 7 show that probes were enriched in the liver, spleen, and kidneys at $1 \mathrm{~h}$ after injection; the enrichment lasted for $4 \mathrm{~h}$, in agreement with the results of $\mathrm{CT}$ imaging. In the spleen, fluorescence intensity began to peak at $1-2 \mathrm{~h}$, which may imply that a large number of macrophages initiated phagocytosis after the injection, that most probes were metabolized at approximately $2 \mathrm{~h}$, and had not accumulated in the spleen $24 \mathrm{~h}$ after injection; this may explain why no spleen uptake was observed at $24 \mathrm{~h}$ post-injection in the optical images. Interestingly, fluorescence imaging of the kidney and liver disagreed with CT imaging of these organs at $24 \mathrm{~h}$, possibly because the sensitivity of the CT imaging system is lower than that of the fluorescence imaging system, and it was difficult to detect the small amount of probes remaining. These results indicate that hybrid $\mathrm{Bi}_{2} \mathrm{~S}_{3}$-QD@DSPE nanoprobes have both CT and fluorescence imaging capability in vivo.

To determine whether $\mathrm{Bi}_{2} \mathrm{~S}_{3}$-QD@DSPE caused any harmful effects or disease, the long-term toxicity of the probes was investigated by monitoring histological changes in several susceptible organs including the heart, lung, spleen, kidney, and liver for more than 2 weeks. Mice were dissected at 1, 3, 7, and 15 days after the injection of a single dose $(0.6 \mathrm{mg} \mathrm{Bi} / \mathrm{g}$ body weight $)$ of $\mathrm{Bi}_{2} \mathrm{~S}_{3}$ QD@DSPE. Hematoxylin and eosin (H\&E) staining of organs showed no significant evidence of tissue damage or adverse effects of the nanoparticles on the organs (Fig. 8). We also measured serum levels of alanine aminotransferase (ALT) and aspartate aminotransferase (AST) over time to determine the effect on liver function. Figure 9 showed that the ALT and AST levels increased transiently after the injection and declined rapidly until they returned to normal. Jain et al. used magnetic nanoparticles in rats and showed similar results [17]. Thus, our probes exhibit little toxicity in the liver. Given that blood can reflect the condition of tissues and organs, we performed blood analysis on mice that received either a single intravenous injection of $150 \mu \mathrm{L}$ phosphate buffered saline (PBS) (control) or $\mathrm{Bi}_{2} \mathrm{~S}_{3}$-QD@DSPE $(0.6 \mathrm{mg} / \mathrm{g})$ at the indicated time. Neither group showed significant differences in any of the following four indexes: white blood cell count (WBC), red blood cell count (RBC), hemoglobin (HGB), or platelet count (PLT), and all indexes were in the reference range (Table 2) [18]. These results further confirmed that $\mathrm{Bi}_{2} \mathrm{~S}_{3}-\mathrm{QD} @ \mathrm{DSPE}$ nanoprobes 

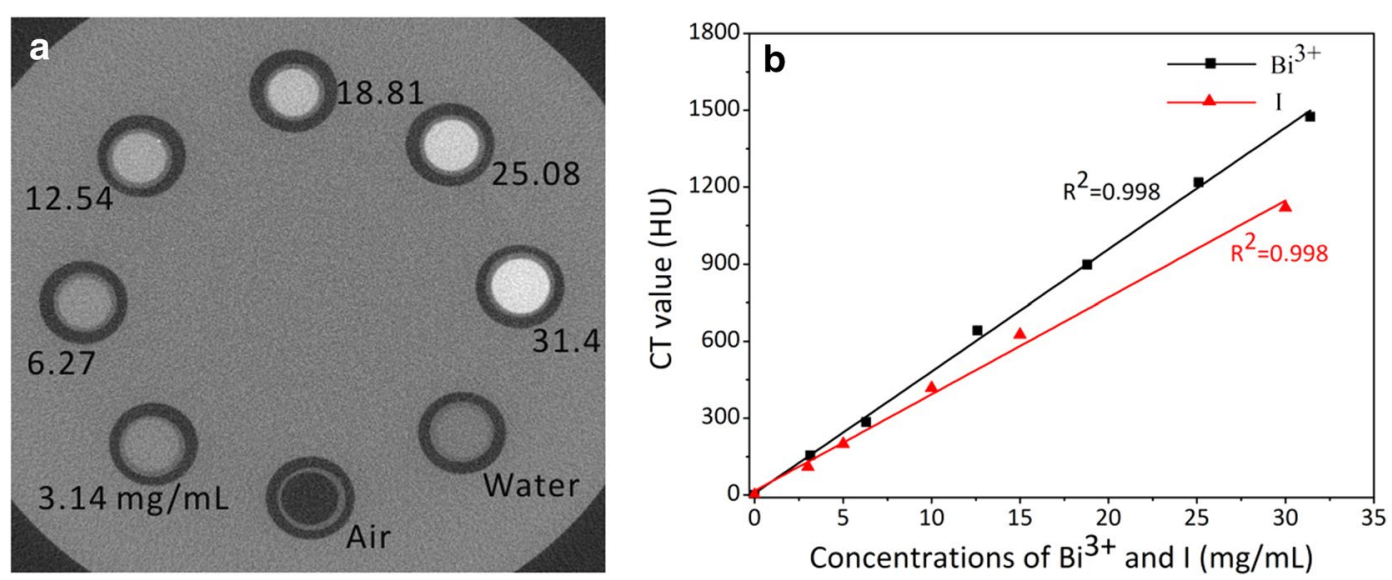

Fig. 4 Phantom images of $\mathrm{Bi}_{2} \mathrm{~S}_{3}$-QD@DSPE hybrid clusters as a function of concentration, using X-ray $\mathrm{CT}$ imaging (a) and HU measurements (b)

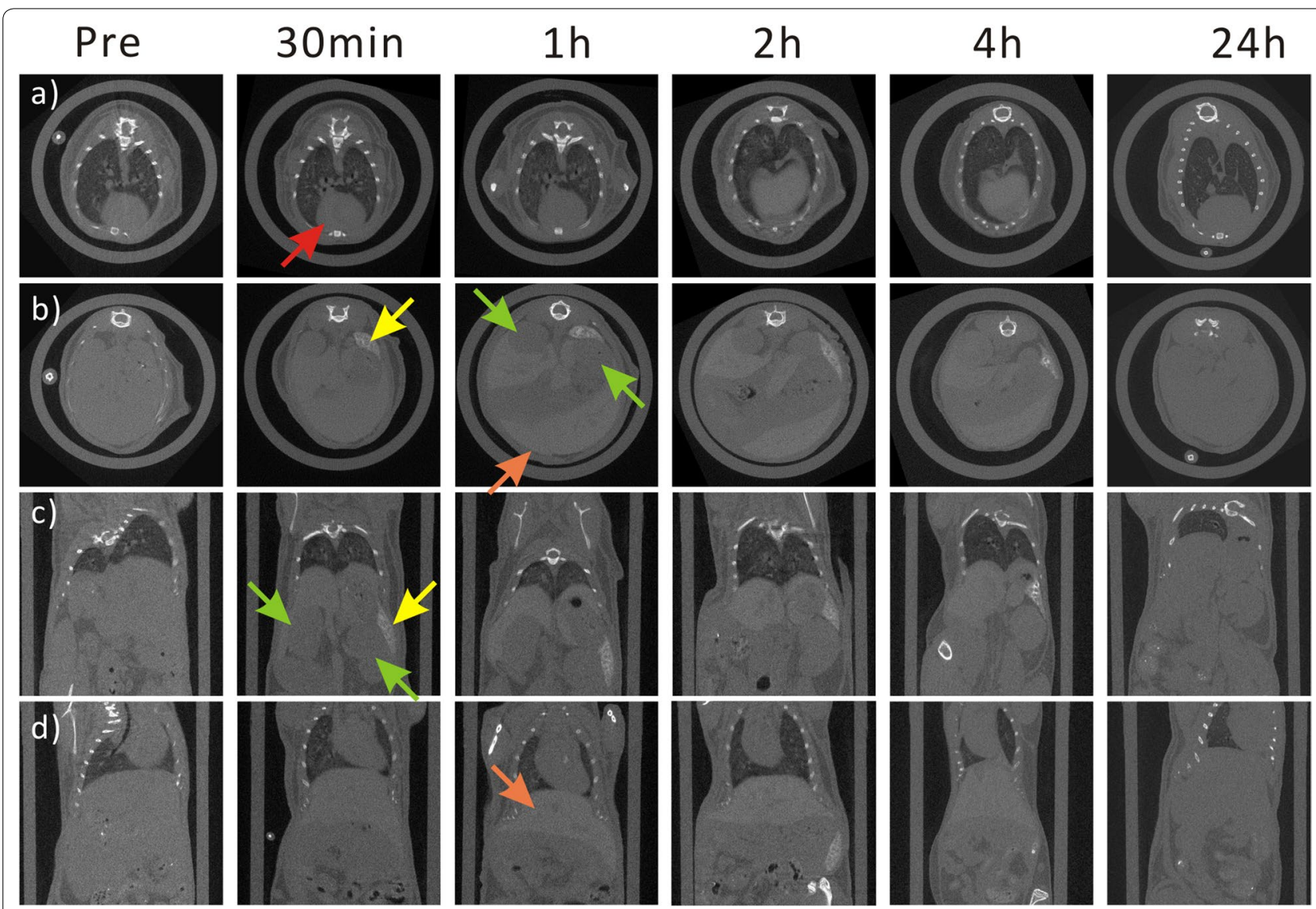

Fig. 5 In vivo X-ray CT imaging. Serial CT sections $(\mathbf{a}, \mathbf{b})$ and coronal views $(\mathbf{c}, \mathbf{d})$ of a mouse at different time points after injection of $\mathrm{Bi}_{2} \mathrm{~S}_{3}-\mathrm{QD} @$ DSPE hybrid clusters in solution ( $0.6 \mathrm{mg} \mathrm{Bi} / \mathrm{g}$ body weight) into the tail vein [heart (a, red arrow), liver (orange arrow), spleen (yellow arrow), and kidney (b, green arrow)] 
Table 1 CT values of the heart, kidney, liver and spleen of mouse before the injection (previous) and at indicated time intervals after the injection

\begin{tabular}{|c|c|c|c|c|c|c|}
\hline & Previous & $30 \mathrm{~min}$ & $1 \mathrm{~h}$ & $2 \mathrm{~h}$ & $4 \mathrm{~h}$ & $24 \mathrm{~h}$ \\
\hline Heart & 81.8 & 196.2 & 123.1 & 81.8 & 91.9 & 102.1 \\
\hline Kidney & 113.0 & 170.9 & 111.5 & 107.2 & 144.1 & 114.4 \\
\hline Liver & 123.1 & 192.6 & 250.5 & 257.1 & 256.3 & 131.8 \\
\hline Spleen & 107.9 & 474.3 & 513.4 & 488.1 & 393.9 & 131.1 \\
\hline
\end{tabular}

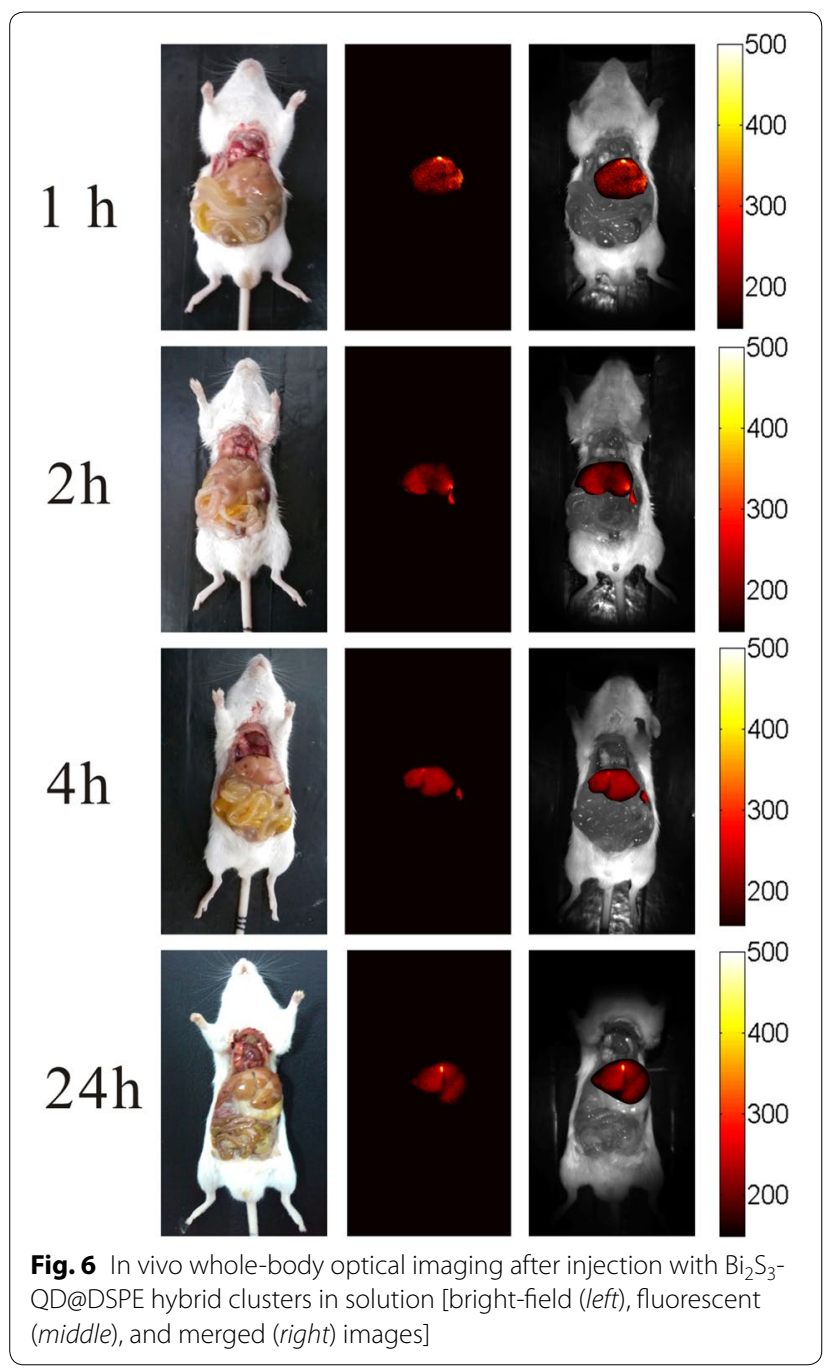

had no significant effect on organs, tissues, or blood, and showed remarkable biocompatibility and promise as a contrast agent.

\section{Conclusion}

In summary, composite core-shell nanoparticles containing $\mathrm{Bi}_{2} \mathrm{~S}_{3}$ nanoparticles and QDs within a single PEGmodified phospholipid were prepared. These nanoprobes exhibit effective dual-mode imaging in vivo, and have the advantages of long circulation time and low toxicity. However, because the excitation wavelength of our in house-built imaging system for the contrast agent probe is $500 \mathrm{~nm}$, which is not conducive to noninvasive optical imaging in vivo, we intend to develop a multi-mode probe that can fully exploit the advantages conferred by the near-infrared range of QDs, in future work.

\section{Methods \\ Materials}

Bismuth neodecanote, oleic acid (90 \%), cadmium oxide (99.99\%), bis(trimethylsilyl) sulfide $\left((\mathrm{TMS})_{2} \mathrm{~S}\right)$, hexadecylamine $(90 \%)$, tri-n-octylphosphine oxide (90\%), and trioctylphosphine (97\%) were purchased from Aldrich. Oleyl amine (90 \%) was purchased from Aladdin. Thioacetamide and selenium powder were purchased from Sinopharm. 1,2-Dipalmitoyl-sn-glycero3-phosphoethanol-amine-N-[methoxy(polyethylene glycol)-2000] (PEG-DSPE, $99 \%$ ) was purchased from Avanti. All chemicals were used as received, unless otherwise stated.

\section{Characterization}

Nanoparticle spectra were measured using a 2550 UVvisible spectrophotometer (Japan) and a LS-55 spectrophotometer (USA). Elemental analysis of the resultant hybrid clusters was performed using an inductively coupled plasma atomic emission spectrometer (Germany). Animal blood analysis was carried out on a SP-4430 dry biochemical analyzer (Japan) and a CA-700 automatic blood analyzer (China). TEM images were captured using a Tecnai G20 U-Twin transmission electron microscope (USA).

\section{Synthesis of $\mathrm{Bi}_{2} \mathrm{~S}_{3}-\mathrm{QD} @ D S P E$}

Based on previously reported methods, we prepared $\mathrm{Bi}_{2} \mathrm{~S}_{3}$ nanoparticles coated with oleic acid [4]. Coreshell $\mathrm{CdSe} / \mathrm{ZnS}$ quantum dots with an emission wavelength of $650 \mathrm{~nm}$ were synthesized using a previously described two-step method [19, 20]. After addition of PEG-DSPE, hydrophobic $\mathrm{Bi}_{2} \mathrm{~S}_{3}$ and $\mathrm{QD}$ were modified and transformed into hydrophilic nanocomposite 


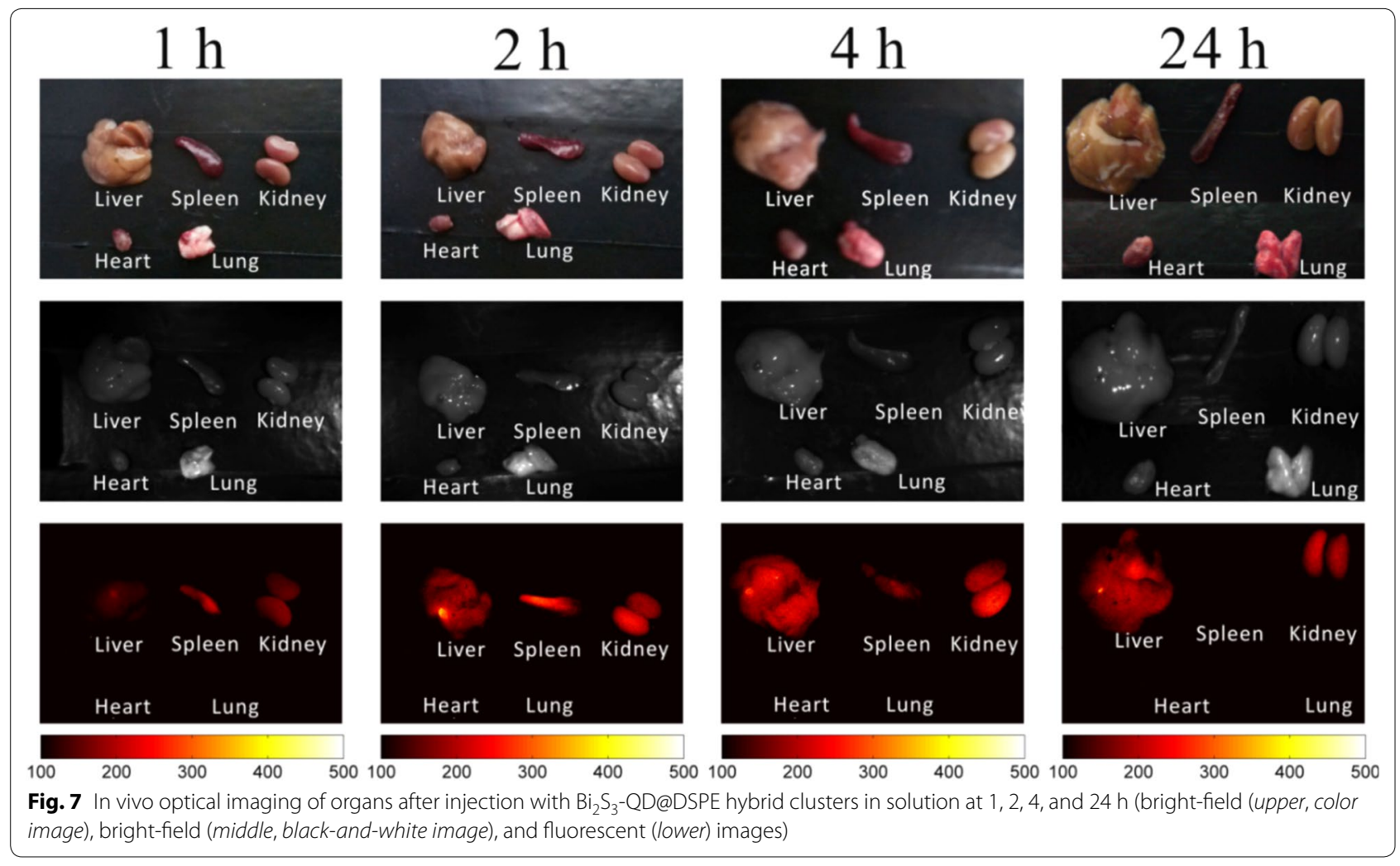

hybrid clusters, $\mathrm{Bi}_{2} \mathrm{~S}_{3}$-QD@DSPE. The main steps were performed according to previously reported methods [13, 21]. In brief, $\mathrm{Bi}_{2} \mathrm{~S}_{3}$ nanoparticles and QD were mixed with PEG-DSPE in chloroform. $\mathrm{Bi}_{2} \mathrm{~S}_{3}$-QD@ DSPE hybrid clusters were prepared by evaporating the organic solvent in a rotary evaporator under vacuum with $300 \mathrm{r} / \mathrm{min}$ rotation speed for $30 \mathrm{~min}$ and then flushing with a $\mathrm{N}_{2}$ stream to remove any residual traces of organic solvent. The lipid film, deposited in the reaction vial, was hydrated with water and subjected to ultrasonication. The resulting dispersion was purified by centrifugation using a Millipore filter (Centrifugal Filter Devices, $100 \mathrm{~K}$ ) to remove excess PEG-DSPE. The final nanoparticles were redispersed in PBS for use in further experiments.

\section{$\mathrm{CT}$ imaging of $\mathrm{Bi}_{2} \mathrm{~S}_{3}-\mathrm{QD} @ \mathrm{DSPE}$}

Various concentrations of $\mathrm{Bi}_{2} \mathrm{~S}_{3}$-QD@DSPE (3.14, 6.27, $12.54,18.81,25.08$, and $31.4 \mathrm{mg}$ of $\mathrm{Bi} / \mathrm{mL}$ ) dispersed in deionized water were prepared in $1.5-\mathrm{mL}$ microtubes. A high-resolution CT system was constructed and applied for imaging, using the following imaging parameters: spatial resolution of $100 \mu \mathrm{m}$, tube voltage of $50 \mathrm{kV}$, and tube current of $0.800 \mathrm{~mA}$.

\section{Measurement, polydispersity index (PDI), and zeta} potential of $\mathrm{Bi}_{2} \mathrm{~S}_{3}-\mathrm{QD} @ D S P E$

The mean particle size, PDI, and zeta potential of $\mathrm{Bi}_{2} \mathrm{~S}_{3}$ QD@DSPE were measured during storage at 4, 25, and $37^{\circ} \mathrm{C}$ using a nano-ZS90 dynamic light scattering (Malvern, UK) according to the manufacturer's instructions.

\section{In vivo $C T$ and fluorescence imaging}

Balb/C mice with an average age of 6-8 weeks were purchased from the Hubei Medical Laboratory Animal Center. All animal studies were approved by the Animal Experimentation Ethics Committee of Huazhong University of Science and Technology. Balb/C mice (male) was anesthetized using $2 \%$ chloral hydrate and $10 \%$ urethane. Subsequently, $\mathrm{Bi}_{2} \mathrm{~S}_{3}$-QD@DSPE (0.6 mg Bi/g body weight) was injected through the tail vein into the mice and CT imaging was performed at appropriate time points (pre-injection, $30 \mathrm{~min}, 1,2$, 4, and $24 \mathrm{~h}$ ) after tail vein injection. The mice were then perfused and cleared with PBS for fluorescence imaging. Experiments were performed in quintuplicate using 5 mice. A high-resolution $\mathrm{CT}$ system was constructed and applied for imaging, using the following imaging parameters: spatial resolution of $100 \mu \mathrm{m}$, tube voltage of $50 \mathrm{kV}$, and tube current of 


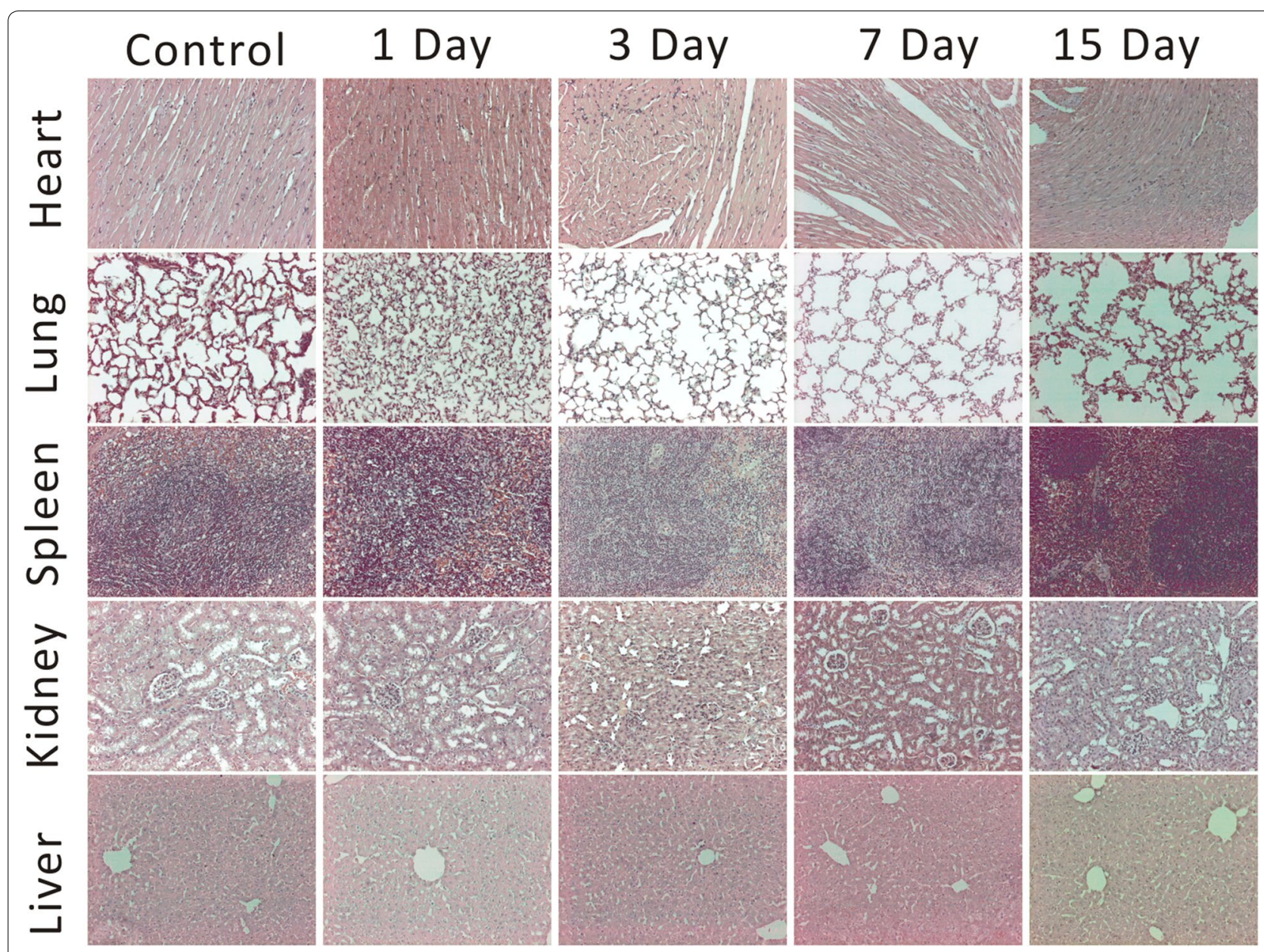

Fig. 8 H\&E-stained tissue sections from mice to observe the time course of the histological changes in the heart, lung, spleen, kidney, and liver tissue of mice receiving a single intravenous injection of PBS (control) or $\mathrm{Bi}_{2} \mathrm{~S}_{3}-\mathrm{QD} @ D S P E$ hybrid clusters solution (0.6 mg Bi/g body weight)

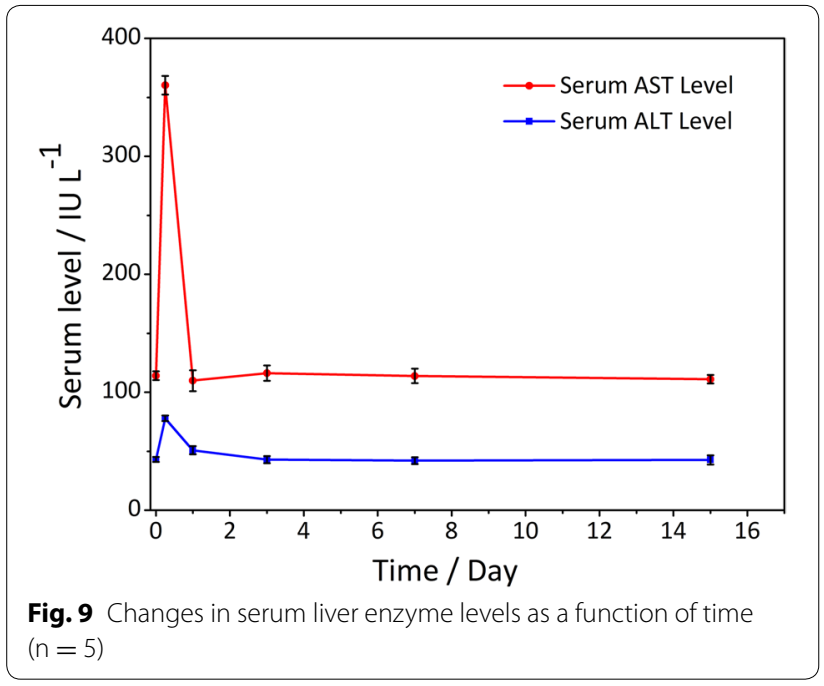

$0.800 \mathrm{~mA}$. Fluorescence images of $\mathrm{Bi}_{2} \mathrm{~S}_{3}-\mathrm{QD} @ \mathrm{DSPE}$ were acquired using a filter set (excitation filter: $469 / 35 \mathrm{~nm}$; emission filter: 655/40 $\mathrm{nm}$ ) and calibrated using an auto-fluorescent background filter set (excitation filter: 396/40 nm; emission filter: 655/40 nm). The CT and fluorescence imaging systems were both built in-house [22].

\section{In vivo safety evaluation of probes}

The male Balb/C mice were randomly assigned to two groups, with five in each group. After receiving a single intravenous injection of either PBS (control) or probes, $250-\mu \mathrm{L}$ blood samples were collected from each mouse by extraction of orbital venous plexus blood, including $200 \mu \mathrm{L}$ of blood for liver function tests (ALT and AST assays, carried out on a SP-4430 dry biochemical analyzer) and $50 \mu \mathrm{L}$ of blood for blood analysis (measured 
Table 2 Blood analysis of mouse that received single intravenous injection of $150 \mu \mathrm{LBS}$ (control) or $\mathrm{Bi}_{2} \mathrm{~S}_{3}-\mathrm{QD} @ \mathrm{DSPE}$ (0.6 $\mathrm{mg} / \mathrm{g}$ dose in PBS) followed by dissection at the indicated times $(n=5)$

\begin{tabular}{|c|c|c|c|c|c|}
\hline & & WBC ${ }^{\mathrm{a}}, 10^{9} / \mathrm{L}$ & $\mathrm{RBC}^{\mathrm{b}}, 10^{12} / \mathrm{L}$ & $\mathrm{HGB}^{\mathrm{c}}, \mathrm{g} / \mathrm{L}$ & $\mathrm{PLT}^{\mathrm{d}}, 10^{9} / \mathrm{L}$ \\
\hline \multirow[t]{2}{*}{ Reference values } & 6 weeks & $7.25-10.13$ & $10.04-11.09$ & $155.57-168.70$ & $992.31-1181.23$ \\
\hline & 8 weeks & $8.08-11.50$ & $10.13-13.19$ & $142.47-188.33$ & $734.60-1270.30$ \\
\hline \multirow[t]{2}{*}{1 day } & PBS & $9.52 \pm 0.74$ & $10.62 \pm 0.20$ & $161.20 \pm 2.86$ & $998.00 \pm 12.59$ \\
\hline & Probe & $9.78 \pm 0.36$ & $10.70 \pm 0.17$ & $160.60 \pm 6.80$ & $1023.00 \pm 50.98$ \\
\hline \multirow[t]{2}{*}{3 day } & PBS & $9.80 \pm 0.45$ & $10.88 \pm 0.18$ & $164.60 \pm 2.70$ & $1022.40 \pm 41.11$ \\
\hline & Probe & $9.84 \pm 0.44$ & $10.75 \pm 0.23$ & $162.40 \pm 7.33$ & $1012.60 \pm 33.09$ \\
\hline \multirow[t]{2}{*}{7 day } & PBS & $9.72 \pm 0.33$ & $10.72 \pm 0.38$ & $165.81 \pm 1.92$ & $1033.40 \pm 34.09$ \\
\hline & Probe & $9.78 \pm 0.26$ & $10.78 \pm 0.36$ & $163.00 \pm 5.34$ & $998.60 \pm 36.67$ \\
\hline \multirow[t]{2}{*}{15 day } & PBS & $10.72 \pm 0.15$ & $11.84 \pm 0.34$ & $172.20 \pm 7.95$ & $970.60 \pm 68.72$ \\
\hline & Probe & $10.46 \pm 0.15$ & $11.92 \pm 0.63$ & $176.20 \pm 6.10$ & $1015.40 \pm 39.17$ \\
\hline
\end{tabular}

\footnotetext{
${ }^{a}$ white blood cell count

b red blood cell count

c hemoglobin

d platelet count
}

using a CA-700 automatic blood analyzer). Organs, including heart, lung, spleen, kidney, and liver were stained with $\mathrm{H} \& \mathrm{E}$ at the appropriate time points $(1,3,7$, and $15 \mathrm{~d})$ and observed under a light microscope at $10 \times$ magnification.

\section{Abbreviations}

CT: computed tomography; QDs: quantum dots; PEG-DSPE: 1,2-dipalmitoylsn-glycero-3-phosphoethanolamine-N-[methoxy (polyethylene glycol)-2000]; PDI: polydispersity index; H\&E: hematoxylin and eosin; ALT: alanine aminotransferase; AST: aspartate aminotransferase; WBC: white blood cell count; RBC: red blood cell count; HGB: hemoglobin; PLT: platelet count; PBS: phosphate buffered saline.

\section{Authors' contributions}

$J C$ performed all experiments except for the imaging experiments. XQY performed the imaging experiments. MYQ helped the Synthesis of probe. XSZ and YX helped the animal studies. YDZ planned all experiments and provided the facilities necessary for the completion of this work. All authors have read and approved the final manuscript.

\section{Author details}

${ }^{1}$ Britton Chance Center for Biomedical Photonics at Wuhan National Laboratory for Optoelectronics - Hubei Bioinformatics \& Molecular Imaging Key Laboratory, Department of Biomedical Engineering, College of Life Science and Technology, Huazhong University of Science and Technology, Wuhan 430074, People's Republic of China. ${ }^{2}$ Key Laboratory of Molecular Biophysics of the Ministry of Education, College of Life Science and Technology, Huazhong University of Science and Technology, Wuhan 430074, People's Republic of China.

\section{Acknowledgements}

This work was supported by the National Natural Science Foundation of China (Grant No. 81271616, 81471697), the Foundation for Innovative Research Groups of the NNSFC (Grant No. 61121004), the Natural Science Foundation of Hubei Province (2014CFB1010), the Key Technology R\&D Program of Hubei Province (2014BBB003) and Yellow Crane Talent (Science and Technology) Program of Wuhan City. We also thank the Analytical and Testing Center (HUST) for the help of measurement.

\section{Competing interests}

The authors declared that they have no competing interests.

Received: 30 July 2015 Accepted: 16 October 2015

Published online: 29 October 2015

\section{References}

1. Li BH, Leung ASO, Soong A, Munding CE, Lee H, Thind AS, Munce NR, Wright GA, Rowsell CH, Yang VXD, Strauss BH, Foster FS, Courtney BK. Hybrid intravascular ultrasound and optical coherence tomography catheter for imaging of coronary atherosclerosis. Catheter Cardio Inte. 2013;81:494-507.

2. Zhu J, Lu Y, Li Y, Jiang J, Cheng L, Liu Z, Guo L, Pan Y, Gu H. Synthesis of $\mathrm{Au}-\mathrm{Fe}_{3} \mathrm{O}_{4}$ heterostructured nanoparticles for in vivo computed tomography and magnetic resonance dual model imaging. Nanoscale. 2014;6:199-202.

3. Kim D, Park S, Lee JH, Jeong YY, Jon S. Antibiofouling polymer-coated gold nanoparticles as a contrast agent for in vivo $x$-ray computed tomography imaging. J Am Chem Soc. 2007;129:7661-5.

4. Ai K, Liu Y, Liu J, Yuan Q, He Y, Lu L. Large-scale synthesis of $\mathrm{Bi}_{2} \mathrm{~S}_{3}$ nanodots as a contrast agent for in vivo $\mathrm{x}$-ray computed tomography imaging. Adv Mater. 2011;23:4886-91

5. Rabin O, Manuel Perez J, Grimm J, Wojtkiewicz G, Weissleder R. An x-ray computed tomography imaging agent based on long-circulating bismuth sulphide nanoparticles. Nat Mater. 2006;5:118-22.

6. Oh MH, Lee N, Kim H, Park SP, Piao Y, Lee J, Jun SW, Moon WK, Choi SH, Hyeon T. Large-scale synthesis of bioinert tantalum oxide nanoparticles for $x$-ray computed tomography imaging and bimodal image-guided sentinel lymph node mapping. J Am Chem Soc. 2011;133:5508-15.

7. Ding JL, Wang YH, Ma M, Zhang Y, Lu SS, Jiang YN, Qi CM, Luo SH, Dong G, Wen S, An YL, Gu N. CT/Fluorescence dual-modal nanoemulsion platform for investigating atherosclerotic plaques. Biomaterials. 2013;34:209-16.

8. Ni D, Bu W, Zhang S, Zheng X, Li M, Xing H, Xiao Q, Liu Y, Hua Y, Zhou L, Peng W, Zhao K, Shi J. Single $\mathrm{Ho}^{3+}$-doped upconversion nanoparticles for high-performance $\mathrm{T}_{2}$-weighted brain tumor diagnosis and MR/UCL/CT multimodal imaging. Adv Funct Mater. 2014;24:6613-20.

9. Xiao Q, Bu W, Ren Q, Zhang S, Xing H, Chen F, Li M, Zheng X, Hua Y, Zhou L, Peng W, Qu H, Wang Z, Zhao K, Shi J. Radiopaque fluorescence-transparent $\mathrm{TaO}_{\mathrm{x}}$ decorated upconversion nanophosphors for in vivo CT/MR/ UCL trimodal imaging. Biomaterials. 2012;33:7530-9. 
10. Xing H, Bu W, Zhang S, Zheng X, Li M, Chen F, He Q, Zhou L, Peng W, Hua $Y$, Shi J. Multifunctional nanoprobes for upconversion fluorescence, MR and CT trimodal imaging. Biomaterials. 2012;33:1079-89.

11. Chou SW, Shau YH, Wu PC, Yang YS, Shieh DB, Chen CC. In vitro and in vivo studies of FePt nanoparticles for dual modal CT/MRI molecular imaging. J Am Chem Soc. 2010;132:13270-8.

12. Chen J, Yang XQ, Meng YZ, Qin MY, Yan DM, QianY XuGQ, YuY Ma ZY, Zhao YD. Reverse microemulsion-mediated synthesis of $\mathrm{Bi}_{2} \mathrm{~S}_{3}-\mathrm{QD} @ \mathrm{SiO}_{2}-$ PEG for dual modal CT-fluorescence imaging in vitro and in vivo. Chem Commun. 2013;49:11800-2.

13. Park J-H, von Maltzahn G, Ruoslahti E, Bhatia SN, Sailor MJ. Micellar hybrid nanoparticles for simultaneous magnetofluorescent imaging and drug delivery. Angew Chem Int Edit. 2008;47:7284-8.

14. Liu Y, Ai K, Liu J, Yuan Q, He Y, Lu L. A high-performance ytterbium-based nanoparticulate contrast agent for in vivo $\mathrm{x}$-ray computed tomography imaging. Angew Chem Int Edit. 2012;51:1437-42.

15. Wang J, Mongayt DA, Lukyanov AN, Levchenko TS, Torchilin VP. Preparation and in vitro synergistic anticancer effect of vitamin $\mathrm{K} 3$ and 18-diazabicyclo[540]undec-7-ene in poly(ethylene glycol)-diacyllipid micelles. Int J Pharm. 2004;272:129-35.
16. Sezgin Z, Yüksel N, Baykara T. Preparation and characterization of polymeric micelles for solubilization of poorly soluble anticancer drugs. Eur Pharm Biopharm. 2006;64:261-8.

17. Jain TK, Reddy MK, Morales MA, Leslie-Pelecky DL, Labhasetwar V. Biodistribution clearance and biocompatibility of iron oxide magnetic nanoparticles in rats. Mol Pharm. 2008;5:316-27.

18. Wang DP, Zeng L, Shang SK. Experimental animals blood physiological and biochemical reference manual. Beijing: Science Press; 2011. p. 18-21.

19. Tian ZQ, Zhang ZL, Gao JH, Huang BH, Xie HY, Xie M, Abruñad HD, Pang DW. Color-tunable fluorescent-magnetic core/shell multifunctional nanocrystals. Chem Commun. 2009;27:4025-7.

20. Wang HQ, Li YQ, Wang JH, Xu Q, Li XQ, Zhao YD. Influence of quantum dot's quantum yield to chemiluminescent resonance energy transfer. Anal Chim Acta. 2008;610:68-73.

21. Dubertret B. In vivo imaging of quantum dots encapsulated in phospholipid micelles. Science. 2002;298:1759-62.

22. Yang $X Q$, Meng $Y Z$, Luo QM, Gong $H$. High resolution in vivo micro-CT with flat panel detector based on amorphous silicon. J X-Ray Sci Technol. 2010;18:381-92.

\section{Submit your next manuscript to BioMed Central and take full advantage of:}

- Convenient online submission

- Thorough peer review

- No space constraints or color figure charges

- Immediate publication on acceptance

- Inclusion in PubMed, CAS, Scopus and Google Scholar

- Research which is freely available for redistribution

Submit your manuscript at

www.biomedcentral.com/submit

C Biomed Central 